\section{MOLECULAR SUBCLASSIFICATION OF VULVAR SQUAMOUS CELL CARCINOMA: PROGNOSTIC SIGNIFICANCE AND REPRODUCIBILITY}

${ }^{1}$ EF Thompson*, ${ }^{2} \mathrm{~L}$ Hoang, ${ }^{3} \mathrm{AK}$ Höhn, ${ }^{4} \mathrm{~A}$ Palicelli, ${ }^{5} \mathrm{KL}$ Talia, ${ }^{6} \mathrm{~N}$ Tchrakian, ${ }^{1} \mathrm{~J}$ Senz, ${ }^{7} \mathrm{~A}$ Jamieson, ${ }^{1} \mathrm{~J}$ Huvila, ${ }^{7} \mathrm{JN}$ Mcalpine, ${ }^{2} \mathrm{CB}$ Gilks, ${ }^{8} \mathrm{M}$ Höckel, ${ }^{9} \mathrm{~N}$ Singh, ${ }^{3} \mathrm{LC}$ Horn. ${ }^{1}$ The University of British Columbia, Molecular Oncology, Vancouver, Canada; ${ }^{2}$ The University of British Columbia, Vancouver General Hospital, Pathology and Laboratory Medicine, Vancouver, Canada; ' ${ }^{3}$ Leipzig University, Division of Gynecologic Pathology, Leipzig, Germany; ${ }^{4}$ Azienda Unit Sanitaria Locale- IRCCS, Pathology Unit, Reggio Emilia, Italy; ${ }^{5}$ Royal Women's Hospital and VCS Foundation, Anatomical Pathology, Parkville, Australia; ${ }^{6}$ Barts Health NHS Trust, London, UK; ${ }^{7}$ The University of British Columbia, Division of Gynaecologic Oncology, Vancouver, Canada; ${ }^{8}$ Leipzig University, Division of Gynecologic Oncology, Department of Obstetrics and Gynecology (Institute of Trier), Leipzig, Germany; ${ }^{9}$ Queen Mary University of London, Barts Health NHS Trust and Centre for Pathology at the Blizard Institute, London, UK

\subsection{6/ijgc-2021-ESG0.616}

Introduction/Background* Vulvar squamous cell carcinoma (VSCC) is subclassified into three prognostically relevant groups: i) HPV-Associated (HPV-A), ii) HPV-Independent TP53 mutated (HPV-I p53abn), and iii) HPV-Independent TP53 wild type (HPV-I p53wt). Immunohistochemistry (IHC) for p16 and p53 are used as surrogate markers for HPV viral integration and TP53 mutational status, respectively.

We assessed the reproducibility of subclassification based on p16 and p53 IHC and evaluated the prognostic significance of VSCC molecular subgroups in a patient cohort treated by vulvar field resection (VFR) surgery.

Methodology VSCC formalin-fixed paraffin-embedded tumour tissue and patient clinicopathologic data from 68 cases treated by vulvar field resection (VFR) were collected from the Leipzig School of Radical Pelvic Surgery. Immunohistochemistry for p16 and p53 was performed at three different institutions and evaluated independently by seven pathologists and two pathology research fellows. Tumors were classified into one of four groups: HPV-A, HPV-I p53wt, HPV-I p53abn, and Indeterminate (requiring additional investigation). Selected cases were further interrogated by HPV RNA in situ hybridisation (ISH) and TP53 sequencing.

Result(s)* Final VSCC subclassification yielded 22 tumors (32.4\%) were HPV-A, 41 (60.3\%) were HPV-I p53abn, and $5(7.3 \%)$ were HPV-I p53wt; the clinicopathologic data is summarised in table 1 . Interobserver agreement (overall Fleiss' kappa statistic) for the four-category classification was 0.74. No statistically significant differences in clinical outcomes between HPV-A and HPV-I VSCC were observed (figure 1).

Conclusion* Interobserver reproducibility of VSCC subclassification based on p16 and p53 IHC is very good, supporting their routine use in clinical practice. VSCCs treated by VFR surgery showed no significant difference in clinical outcomes when stratified based on HPV status. This contrasts with the significant prognostic differences between HPV-A and HPV-I VSCC demonstrated in cohorts treated by conventional conservative surgical approaches and suggests that a change in surgical approach can improve outcomes in patients with HPV-I VSCC.

Abstract 231 Table 1 Clinicopathologic features of vulvar squamous cell carcinoma stratified by HPV status

\begin{tabular}{|c|c|c|c|c|c|c|c|c|c|c|}
\hline & \multirow{3}{*}{$\begin{array}{c}\text { Variable } \\
\text { Total }\end{array}$} & \multirow{2}{*}{\multicolumn{2}{|c|}{ Total }} & \multirow{2}{*}{\multicolumn{2}{|c|}{ HPV-associated }} & \multicolumn{4}{|c|}{ HPV-independent } & \multirow[b]{3}{*}{$p$} \\
\hline & & & & & & \multicolumn{2}{|c|}{ TP53 wild-type } & \multicolumn{2}{|c|}{ TP53 mutated } & \\
\hline & & (n) & $(\%)$ & (n) & (\%) & (n) & (\%) & (n) & (\%) & \\
\hline & & 68 & $100.0 \%$ & 22 & $32.4 \%$ & 5 & $7.4 \%$ & 41 & $60.3 \%$ & \\
\hline \multicolumn{2}{|c|}{ Follow-up (years, median) } & 2.2 & & 3.5 & & 3.6 & & 1.7 & & \\
\hline \multicolumn{2}{|c|}{ Age [years, median, (SD)] } & 66.1 & 15.2 & 60.5 & 16.0 & 77.0 & 7.9 & 74.0 & 14.1 & 0.01 \\
\hline \multirow[t]{4}{*}{ Stage } & 1 & 35 & $51.5 \%$ & 10 & $45.5 \%$ & 4 & $80.0 \%$ & 21 & $51.2 \%$ & 0.057 \\
\hline & II & 4 & $5.9 \%$ & 4 & $18.2 \%$ & 0 & & 0 & $0.0 \%$ & \\
\hline & III & 26 & $38.2 \%$ & 6 & $27.3 \%$ & 1 & $20.0 \%$ & 19 & $46.3 \%$ & \\
\hline & IV & 3 & $4.4 \%$ & 2 & $9.1 \%$ & 0 & & 1 & $2.4 \%$ & \\
\hline \multirow[t]{3}{*}{ Grade } & 1 & 10 & $14.7 \%$ & 3 & $13.6 \%$ & 4 & $80.0 \%$ & 3 & $7.3 \%$ & $<0.001$ \\
\hline & 2 & 31 & $45.6 \%$ & 14 & $63.6 \%$ & 1 & $20.0 \%$ & 16 & $39.0 \%$ & \\
\hline & 3 & 27 & $39.7 \%$ & 5 & $22.7 \%$ & 0 & & 22 & $53.7 \%$ & \\
\hline \multicolumn{2}{|c|}{ Tumour size $[\mathrm{cm}$, median (SD)] } & 3.3 & 2.2 & 2.7 & 2.0 & 1.5 & 2.2 & 3.5 & 2.2 & 0.35 \\
\hline \multicolumn{2}{|l|}{ LVI } & 40 & $58.8 \%$ & 11 & $50.0 \%$ & 2 & $40.0 \%$ & 27 & $65.9 \%$ & 0.36 \\
\hline \multicolumn{2}{|c|}{ Positive lymph node(s) ${ }^{a}$} & 29 & $42.6 \%$ & 8 & $36.4 \%$ & 1 & $20.0 \%$ & 20 & $48.8 \%$ & 0.65 \\
\hline \multicolumn{2}{|c|}{ Distant Metastases } & 3 & $4.4 \%$ & 2 & $9.1 \%$ & 0 & & 1 & $2.4 \%$ & 0.58 \\
\hline \multicolumn{2}{|c|}{ Adjuvant radiotherapy } & 2 & $2.9 \%$ & 1 & $4.5 \%$ & 0 & & 1 & $2.4 \%$ & 1 \\
\hline \multicolumn{2}{|c|}{ Died of disease } & 11 & $16.2 \%$ & 2 & $9.1 \%$ & 1 & $20.0 \%$ & 8 & $19.5 \%$ & 0.63 \\
\hline \multicolumn{2}{|c|}{ Recurrent disease } & 9 & $13.2 \%$ & 2 & $9.1 \%$ & 1 & $20.0 \%$ & 6 & $14.6 \%$ & 1 \\
\hline
\end{tabular}


Abstract 231 Table 2 Summary of the clinicopathologic features by ProMisE molecular subtype

\begin{tabular}{|c|c|c|c|c|c|}
\hline Variable & Total & POLEmut & MMRd & NSMP & p53abn \\
\hline Total & 172 & 21 (12.2\%) & $47(27.3 \%)$ & 74 (43.0\%) & 30 (17.4\%) \\
\hline $\begin{array}{l}\text { Age at diagnosis } \\
<60 \text { years } \\
\geq 60 \text { years }\end{array}$ & $\begin{array}{c}70(40.7 \%) \\
102(59.3 \%)\end{array}$ & $\begin{array}{c}12(57.1 \%) \\
9(42.9 \%)\end{array}$ & $\begin{array}{l}18(38.3 \%) \\
29(61.7 \%)\end{array}$ & $\begin{array}{l}38(51.4 \%) \\
36(48.6 \%)\end{array}$ & $\begin{array}{c}2(6.6 \%) \\
28(93.3 \%)\end{array}$ \\
\hline $\begin{array}{l}\text { Histotype } \\
\text { Endometrioid } \\
\text { Non-endometrioid } \\
\text {-Serous } \\
\text {-Clear cell } \\
\text {-Carcinosarcoma } \\
\text {-Mixed }\end{array}$ & $\begin{array}{c}137(79.7 \%) \\
35(20.3 \%) \\
15(8.7 \%) \\
2(1.2 \%) \\
8(4.7 \%) \\
10(5.8 \%)\end{array}$ & $\begin{array}{l}18(85.7 \%) \\
3(14.3 \%) \\
0 \\
0 \\
0 \\
3(14.3 \%)\end{array}$ & $\begin{array}{l}45(95.7 \%) \\
2(4.3 \%) \\
1(2.1 \%) \\
0 \\
0 \\
1(2.1 \%)\end{array}$ & $\begin{array}{l}71(95.9 \%) \\
3(4.1 \%) \\
0 \\
1(1.4 \%) \\
0 \\
2(2.7 \%)\end{array}$ & $\begin{array}{l}3(10.0 \%) \\
27(90.0 \%) \\
14(46.7 \%) \\
1(3.3 \%) \\
8(26.7 \%) \\
4(13.3 \%)\end{array}$ \\
\hline $\begin{array}{l}\text { Tumour grade } \\
\text { low grade } \\
\text { high grade }\end{array}$ & $\begin{array}{c}129(75 \%) \\
43(25 \%)\end{array}$ & $\begin{array}{c}16(76.2 \%) \\
5(23.8 \%)\end{array}$ & $\begin{array}{c}41(87.2 \%) \\
6(12.8 \%)\end{array}$ & $\begin{array}{c}69(93.2 \%) \\
5(6.8 \%)\end{array}$ & $\begin{array}{c}2(6.7 \%) \\
28(93.3 \%)\end{array}$ \\
\hline $\begin{array}{l}\text { Tumour size } \\
<2 \mathrm{~cm} \\
\geq 2 \mathrm{~cm} \\
\text { unk }\end{array}$ & $\begin{array}{c}31(18.0 \%) \\
132(76.8 \%) \\
9(5.2 \%)\end{array}$ & $\begin{array}{c}4(19.0 \%) \\
17(81.0 \%) \\
0\end{array}$ & $\begin{array}{c}12(25.5 \%) \\
35(74.5 \%) \\
0\end{array}$ & $\begin{array}{c}14(18.9 \%) \\
53(71.6 \%) \\
7(9.5 \%)\end{array}$ & $\begin{array}{c}1(3.3 \%) \\
27(90.0 \%) \\
2(6.7 \%)\end{array}$ \\
\hline $\begin{array}{l}\text { Myoinvasion } \\
\text { None } \\
<50 \% \\
\geq 50 \%\end{array}$ & $\begin{array}{l}39(22.7 \%) \\
82(47.7 \%) \\
51(29.6 \%)\end{array}$ & $\begin{array}{c}5(23.8 \%) \\
11(52.4 \%) \\
5(23.8 \%)\end{array}$ & $\begin{array}{l}13(27.7 \%) \\
19(40.4 \%) \\
15(31.9 \%)\end{array}$ & $\begin{array}{l}16(21.6 \%) \\
37(50.0 \%) \\
21(28.4 \%)\end{array}$ & $\begin{array}{c}5(16.7 \%) \\
15(50 \%) \\
10(33.3 \%)\end{array}$ \\
\hline $\begin{array}{l}\text { FIGO stage } \\
\text { I-II } \\
\text { III-IV }\end{array}$ & $\begin{array}{l}140(81.4) \\
32(18.6 \%)\end{array}$ & $\begin{array}{c}17(81.0 \%) \\
4(19.0 \%)\end{array}$ & $\begin{array}{c}43(91.5 \%) \\
4(8.5 \%)\end{array}$ & $\begin{array}{l}64(86.5 \%) \\
10(13.5 \%)\end{array}$ & $\begin{array}{l}15(50.0 \%) \\
15(50.0 \%)\end{array}$ \\
\hline $\begin{array}{l}\text { LVI } \\
\text { Negative } \\
\text { Positive }\end{array}$ & $\begin{array}{c}117(68.0 \%) \\
55(32.0 \%)\end{array}$ & $\begin{array}{c}15(71.4 \%) \\
6(28.6 \%)\end{array}$ & $\begin{array}{l}31(66.0 \%) \\
16(34.0 \%)\end{array}$ & $\begin{array}{l}59(79.7 \%) \\
15(20.3 \%)\end{array}$ & $\begin{array}{l}12(40.0 \%) \\
18(60.0 \%)\end{array}$ \\
\hline $\begin{array}{l}\text { Pelvic and Para- } \\
\text { aortic LND } \\
\text { Yes } \\
\text { No }\end{array}$ & $\begin{array}{c}171(99.4 \%) \\
1(0.6 \%)\end{array}$ & 21 (100\%) & 47 (100\%) & 74 (100\%) & $\begin{array}{c}29(96.7 \%) \\
1(3.3 \%)\end{array}$ \\
\hline $\begin{array}{l}\text { Presence of } L N \\
\text { metastases }\end{array}$ & 31 (18.1\%) & $3(14.2 \%)$ & 7 (14.9\%) & $8(10.8 \%)$ & $13(44.8 \%)$ \\
\hline
\end{tabular}

LN, Iymph node; LND, lymph node dissection; LVI, Iymphovascular invasion; unk, unknown. 